\title{
House dust mite induces Sonic hedgehog signaling that mediates epithelial-mesenchymal transition in human bronchial epithelial cells
}

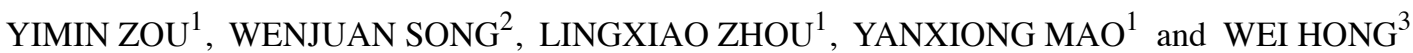 \\ ${ }^{1}$ Key Laboratory of Respiratory Disease of Zhejiang Province, Department of Respiratory and Critical Care Medicine, \\ The Second Affiliated Hospital, Zhejiang University School of Medicine, Hangzhou, Zhejiang 310009; \\ ${ }^{2}$ Department of Economics, School of Economics and Management, Zhejiang Sci-Tech University, \\ Hangzhou, Zhejiang 310018; ${ }^{3}$ Biological Laboratory Center, Guangzhou Institute of \\ Biomedicine and Health Joint School of Life Sciences, Guangzhou Medical University, \\ Guangzhou, Guangdong 510030, P.R. China
}

Received May 2, 2019; Accepted September 9, 2019

DOI: $10.3892 / \mathrm{mmr} .2019 .10707$

\begin{abstract}
Epithelial-mesenchymal transition (EMT) provides a valuable source of fibroblasts that produce extracellular matrix in airway walls. The Sonic hedgehog ( $\mathrm{SHH})$ signaling pathway plays an essential role in regulating tissue turnover and homeostasis. SHH is strikingly upregulated in the bronchial epithelia during asthma. Snaill is a major target of $\mathrm{SHH}$ signaling, which regulates EMT and fibroblast motility. The present study was designed to ascertain whether the combination of house dust mite (HDM) and transforming growth factor $\beta 1$ (TGF- $\beta 1$ ) could induce EMT via the SHH signaling pathway in human bronchial epithelial cells (HBECs). HBEC cultures were treated with HDM/TGF- $\beta 1$ for different periods of time. The involvement of SHH signaling and EMT biomarkers was evaluated by quantitative real-time PCR, western blotting and immunofluorescence staining. Small-interfering RNA (siRNA) for glioma-associated antigen-1 (Gli1) or cyclopamine was used to inhibit SHH signaling in HBECs. HBECs stimulated by HDM/TGF- $\beta 1$ exhibited morphological features of EMT. E-cadherin (an epithelial marker) was decreased after a $72-\mathrm{h}$ exposure to HDM/TGF- $\beta 1$ compared to that in the control cells, and the expression of type I collagen and FSP1 (mesenchymal markers) was increased. HDM/TGF- $\beta 1$ activated the SHH signaling pathway in HBECs, which led to Gli1 nuclear translocation and the transcriptional activation of Snaill expression. Moreover, gene silencing or the pharma-
\end{abstract}

Correspondence to: Dr Wei Hong, Biological Laboratory Center, Guangzhou Institute of Biomedicine and Health Joint School of Life Sciences, Guangzhou Medical University, 195 West Dongfeng Road, Yuexiu, Guangzhou, Guangdong 510030, P.R. China

E-mail: weihong5566@sina.com

Key words: epithelial-mesenchymal transition, bronchial epithelial cells, house dust mite, Sonic hedgehog signaling cological inhibition of Gli1 ameliorated EMT. In summary, these findings suggest that HDM/TGF- $\beta 1$ may induce EMT in $\mathrm{HBEC}$ via an $\mathrm{SHH}$ signaling mechanism. Inhibition of $\mathrm{SHH}$ signaling may be a novel therapeutic method for preventing airway remodeling in asthma.

\section{Introduction}

Asthma is a complex type of allergic inflammation that principally involves the conducting airways and is often related to airway remodeling (1). The importance of tissue remodeling, which involves extracellular matrix (ECM) deposition and fibroblast proliferation instead of eosinophilic infiltration in the airway walls, is an early and persistent component of asthma that has been emphasized (2). Fibroblasts may play a key role in peribronchial fibrosis. Nevertheless, the origin of these fibroblasts and the exact pathogenesis of airway remodeling in asthma remain unclear.

The destruction of epithelial integrity is an important event in airway remodeling and airway hyperresponsiveness (3). In recent years, studies have suggested that epithelial-mesenchymal transition (EMT) provides a direct source of fibroblasts (4-6). EMT is a biological process in which epithelial cells undergo multiple biochemical changes to acquire a mesenchymal cell phenotype. House dust mite (HDM) is the major indoor allergen and is associated with allergic response in asthma patients. A previous study demonstrated that HDM combined with transforming growth factor $\beta 1$ (TGF- $\beta 1$ ) can induce marked EMT characteristics (7). Chronic HDM exposure leads to TGF- $\beta$ expression in the airway epithelium and the induction of EMT (8). These results suggest that EMT may play a unique role in the airway remodeling of asthma. However, the exact molecular mechanism of EMT in allergic asthma remains unclear.

Sonic hedgehog ( $\mathrm{SHH}$ ) signaling is an evolutionarily conserved pathway involved in a variety of biological processes during normal embryonic development and adult tissue homeostasis (9). SHH signaling molecules include a 
receptor Patched (Ptch) and a signal transducer Smoothened (Smo). Once Hedgehog binds to Ptch, the inhibition of Smo is relieved, leading to the translocation of full-length Gli proteins into the nucleus and the activation of the expression of hedgehog target genes (10). The Sonic hedgehog pathway is significantly upregulated in the airway epithelium of children with asthma (11). Snaill is a major target of the SHH signaling pathway, which regulates EMT and fibroblast motility (12). Shh is expressed in the lung and regulates epithelial-mesenchymal crosstalk (13). A genome-wide association study linked Hedgehog interacting protein (HHIP) and Ptch1 mutations to lung function decline and different asthma phenotypes (14). HHIP acts in a negative feedback loop to attenuate Hedgehog signaling by affecting Hedgehog proteins. However, the mechanism of SHH signaling that contributes to the pathophysiology of asthma is still unclear.

To the best of our knowledge, there has been no systematic study of the possible molecular mechanisms of HDM that may be involved in EMT in human bronchial epithelial cells (HBECs). A previous study demonstrated that HDM alone could not induce cell morphological and phenotypic changes in HBECs, but HDM combined with TGF- $\beta 1$ can induce marked EMT characteristics (7). Therefore, the present study was designed to examine whether HDM/TGF- $\beta 1$ could trigger EMT in HBECs via the SHH signaling pathway. The identification of the exact molecular mechanism that underlies EMT may facilitate the search for new targets to prevent airway remodeling in asthma.

\section{Materials and methods}

Cell culture. An HBEC cell line (16HBECs; American Type Culture Collection/ATCC) was cultured in $2 \mathrm{ml}$ of DMEM (Sigma-Aldrich; Merck KGaA) containing 10\% foetal bovine serum (FBS) and penicillin $(100 \mathrm{U} / \mathrm{ml})$. Prior to treatment, cells were seeded at a density of $5-6 \times 10^{5}$ cells per well in 6 -well plates. The cells were maintained at $37^{\circ} \mathrm{C}$ in a humidified atmosphere with $5 \% \mathrm{CO}_{2}$. The confluent $16 \mathrm{HBECs}$ were serum-starved overnight and were incubated with or without HDM (30 $\mu \mathrm{g} / \mathrm{ml}$; cat. no. XPB82D3A2.5; Greer Laboratories, Lenoir, NC, USA) and TGF- $\beta 1$ (5 ng/ml; cat. no. T7039; Sigma-Aldrich; Merck KGaA) for 24-72 h.

Cell viability assay. To determine a suitable concentration for cyclopamine treatment, a Counting Kit-8 assay (CCK-8) was used to monitor cellular viability. In brief, 16HBECs were seeded at a density $1 \times 10^{3}$ cells $/ \mathrm{ml}$ in 96 -well plates and were treated with cyclopamine at different concentrations $(0,5,10,20$ and $40 \mu \mathrm{M})$ for $72 \mathrm{~h}$. At the end of the treatment, CCK-8 reagent (Dojindo Molecular Technologies, Inc., Kumamoto, Japan) was added to each well of the plates, and then the plates were incubated at $37^{\circ} \mathrm{C}$ in an incubator for $4 \mathrm{~h}$. Finally, the absorbance values at $450 \mathrm{~nm}$ were measured using a microplate reader (FLX800TBID, Bio-Tek Instruments, USA). The cell viability was analyzed in triplicate.

Western blot analysis. The cells were lysed on ice using RIPA buffer containing protease inhibitors. The cytoplasmic extract (CE) buffer was composed of $1 \%$ Nonidet P-40, $150 \mathrm{mM} \mathrm{NaCl}$, $50 \mathrm{mM}$ Tris $\mathrm{pH} 8.0,1 \mathrm{mM}$ sodium orthovanadate and $5 \mathrm{mM}$
$\mathrm{NaF}$. The nuclear and cytoplasmic proteins were extracted according to the manufacturer's protocol, and proteins (30 $\mu \mathrm{g} /$ lane) were separated by $10 \%$ SDS-PAGE, subsequently electro-transferred onto PVDF membranes. The detection of blotted proteins was performed with anti-E-cadherin (1:500; cat. no. sc-8426), anti-type I collagen (1:1,000; cat. no. sc-59772), anti-Gli1 (1:1,000; cat. no. sc-20687), anti-Snail1 (1:1,000; cat. no. sc-271977), anti-GAPDH (1:1,000; cat. no. sc-47724) (Santa Cruz Biotechnology, Inc.) and anti-FSP1 (1:1,000; cat. no. ab124805; Abcam) antibodies. Primary antibodies were added to the membrane in 5\% nonfat dry milk at $4{ }^{\circ} \mathrm{C}$ overnight and were then incubated with a horseradish peroxidase-linked anti-rabbit or anti-mouse secondary antibody (1:2,000; cat. no. sc-2004, sc-2005; Santa Cruz Biotechnology, Inc.) for $1 \mathrm{~h}$ at room temperature. Immunodetection was performed by chemiluminescence (ECL, Millipore, Billerica, MA, USA). GAPDH was used as an internal control. The gray levels of the blots were measured using Image J software version 1.48 (National Institutes of Health, Bethesda, MD, USA). The experiment was repeated three times.

Immunofluorescence staining. Immunofluorescence staining was performed as described previously (15). HDM and TGF- $\beta 1$ were added to the wells. The cells were incubated with anti-E-cadherin (1:50; cat. no. sc-8426), anti-Glil (dilution 1:100; cat. no. sc-20687; Santa Cruz Biotechnology, Inc.), and anti-FSP1 (1:150; cat. no. ab124805; Abcam) antibodies at $4^{\circ} \mathrm{C}$ overnight, and were subsequently stained with secondary antibodies conjugated with Alexa Green 488 (1:1,000; cat. no. A-11001; Molecular Probes) or with Cy3-labelled secondary antibodies (1:1,000; cat. no. 111-136-144; Jackson ImmunoResearch Laboratories) for $1 \mathrm{~h}$ at room temperature. Cells were also stained with DAPI. The slides were visualized with a Zeiss Axio Imager 2 microscope (Carl Zeiss AG).

Reverse transcription-quantitative $(R T-q) P C R$. Total RNA was isolated and reverse transcribed into cDNA using a ReverTra Ace qPCR RT kit (Toyobo Biotechnology, Tokyo, Japan). Gene expression was determined by SYBR Green Real-Time PCR Master Mix (Toyobo Biotechnology). The primer pairs were synthesized by Invitrogen; Thermo Fisher Scientific, Inc. The primer sequences used were as follows: SHH (sense 5'-AAGGTATGAAGGGAAGATCT-3' and antisense 5'-CCAAAGCGTTCAACTTGT C-3'); Gli1 (sense 5'-GTGGAAATGACTGGCAATGC-3' and antisense 5'-TGCGGCGTTCAAGAGAGACT-3'); Snail1 (sense 5'-TCCTTCGTCCTTCTCCTCT A-3' and antisense 5'-GCT TCGGATGTGCATCTTG-3') and GAPDH (sense 5'-GCC TTCCGTGCCCCACTGC-3' and antisense 5'-GGCTGG TGGTCCAGGGGTCT-3'). GAPDH was used as an internal control. Gene expression was calculated using the $2^{-\Delta \Delta \mathrm{Cq}}$ method (16).

Blockade of SHH signaling. Small-interfering RNA (siRNA) for Glil or cyclopamine was used to inhibit SHH signaling. Cyclopamine is an isolated alkaloid that shows strong potential to bind to SMO and inhibit the SHH signaling pathway (10). Cells (16HBECs) were seeded in 24-well plates at a density of $3 \times 10^{4}$ cells/well and were transfected with siRNA for Gli1 
(5'-AACUCCACAGGCAUACAGGAU-3') and a negative control siRNA (5'-AACGUACGCGGAAUACAACGA-3') purchased from Sangon Biotech (Shanghai, China), which were used in a previous study (17). siRNA was introduced into cells using Lipofectamine LIXV reagent (Invitrogen; Thermo Fisher Scientific, Inc.). Twenty-four hours later, the cells were stimulated with HDM/TGF- $\beta 1$. After $72 \mathrm{~h}$, cells were harvested for the detection of downstream target genes in the SHH signaling pathway. All of the siRNA experiments were performed in triplicate.

Cyclopamine (Sigma-Aldrich; Merck KGaA) was dissolved in dimethyl sulfoxide (DMSO) (at $100 \mathrm{mmol} / \mathrm{l}$ stock solution) (18). The cells were transfected in 24-well plates at a density of $3 \times 10^{4}$ cells/well. After $24 \mathrm{~h}$, the 16 HBECs were stimulated with HDM/TGF- $\beta 1$ and were then treated with cyclopamine for $72 \mathrm{~h}$. Control wells were treated with only $0.1 \%(\mathrm{v} / \mathrm{v})$ DMSO. The total amount of DMSO in the medium never exceeded $1 \%(\mathrm{v} / \mathrm{v})$. Finally, the cells were harvested for further experiments.

Statistical analysis. All data are expressed as the mean \pm the standard error of the mean (SEM). Group differences were analyzed by a Student's t-test or one-way ANOVA with Tukey post hoc analysis. Statistical significance was set at $\mathrm{P}<0.05$.

\section{Results}

HDM combined with TGF- $\beta 1$ induces EMT in HBECs. The HDM and TGF- $\beta 1$ concentrations were chosen according to previous studies $(7,19)$. First, we examined whether HDM/TGF- $\beta 1$ could induce a morphological change that was characteristic of EMT. Cells (16HBECs) in culture exhibited a cobblestone morphology. However, after stimulation by HDM/TGF- $\beta 1$, the cells acquired an elongated and mesenchymal-like morphology with a loss of cell-cell contact (Fig. 1A). After $72 \mathrm{~h}$ of exposure to HDM/TGF- $\beta 1$, western blot analysis demonstrated that the protein level of E-cadherin as decreased; however, the expression levels of mesenchymal markers type I collagen and FSP1 were markedly upregulated compared to that in the controls (Fig. 1C and D). As shown by immunofluorescence staining, the expression of E-cadherin was decreased in the 16HBECs after $72 \mathrm{~h}$ of treatment with HDM/TGF- $\beta 1$, and the expression of FSP1 was increased compared to that in the controls (Fig. 1E). These results indicated that HDM/TGF- $\beta 1$ induces EMT characteristics in the HBECs.

HDM combined with TGF- $\beta 1$ induces an increase in the expression of SHH signaling in HBECs. As shown by western blotting, the cells exposed to HDM/TGF- $\beta 1$ for $24 \mathrm{~h}$ exhibited slightly increased Shh secretion compared to that in the control cells, and this difference gradually increased when cells were exposed for $72 \mathrm{~h}$ (Fig. 2A and B). qPCR data demonstrated a significant increase in Shh mRNA expression after $48 \mathrm{~h}$ of HDM/TGF- $\beta 1$ treatment (Fig. $2 \mathrm{C}$ ). These findings demonstrated that the gene expression and protein levels of Shh were increased. Since Shh has repeatedly been shown to activate SHH-Gli1 signaling in vitro $(20,21)$, we explored whether HDM/TGF- $\beta 1$ could induce an accumulation of Gli1.
Indeed, exposure to HDM/TGF- $\beta 1$ for $24 \mathrm{~h}$ resulted in an accumulation of the total Glil levels compared to the control (Fig. 2C). Snaill is a well-known target of SHH signaling that regulates EMT and fibroblast motility (12). The expression of Snaill was significantly increased in the 16HBECs exposed to $\mathrm{HDM} / \mathrm{TGF}-\beta 1$ for $48 \mathrm{~h}$ compared with its expression in the control cells (Fig. 2A-C).

Blockade of SHH signaling inhibits the nuclear translocation of Glil. The nuclear translocation of Gli1 is a key inducer of Snail1 protein expression (22). The nuclear localization and accumulation of Gli1 are necessary for its transcriptional activity. By immunofluorescence staining, we found that Gli1 protein expression was increased in cells that were treated with HDM/TGF- $\beta 1$ for $72 \mathrm{~h}$ compared to that in untreated cells and was mainly localized in the nucleus of the HDM/TGF- $\beta 1$-stimulated 16HBECs (Fig. 3A and B).

Using a CCK-8 assay, cyclopamine treatment was shown to lead to the inhibition of 16HBEC survival in a dose-dependent manner (Fig. 1B). In the present study, cyclopamine $(10 \mu \mathrm{M})$ was used to specifically block the SHH signaling pathway. After silencing of Glil using siRNA or cyclopamine, the nuclear translocation and protein expression of Gli1 were significantly decreased (Figs. 3A and B, 4A-C).

Inhibition of SHH signaling attenuates HDM/TGF- $\beta 1$-induced EMT in 16HBECs. To examine whether SHH signaling mediates HDM/TGF- $\beta 1$-induced EMT, siRNA was used to silence Gli1. Gli1 siRNA was transfected into 16HBECs and reduced Gli1 protein expression in 16HBECs that were treated or untreated by HDM/TGF- $\beta 1$. Compared to transfection with a negative control siRNA, transfection with Gli1-specific siRNA significantly decreased the EMT process, as demonstrated by the decreased expression of type I collagen and FSP1 and the increased expression of E-cadherin (Fig. 4A and B). Cultured 16HBECs were treated with cyclopamine following HDM/TGF- $\beta 1$ stimulation. As shown in Fig. $4 \mathrm{~A}$ and C, this inhibitor of SHH signaling effectively antagonized the EMT process in vitro.

\section{Discussion}

The bronchial epithelium is an initial barrier and may be more susceptible to inhaled noxious agents (7). Airway remodeling is a marked feature of asthma that aims to maintain epithelial integrity. The present study revealed that in human bronchial epithelial cells (HBECs), exposure to HDM/TGF- $\beta 1$-induced epithelial-mesenchymal transition (EMT), which was identified by the upregulation of mesenchymal markers (FSP1 and type I collagen) and the loss of the adherens junction protein E-cadherin. We believe these findings provide evidence for the upregulation of the Sonic hedgehog ( $\mathrm{SHH}$ ) signaling pathway in house dust mite (HDM)/transforming growth factor $\beta 1$ (TGF- $\beta 1$ )-mediated EMT in HBECs. Furthermore, SHH activated the transcription factor Snaill and was sufficient to induce matrix production in HBECs in vitro. These results demonstrated that HDM/TGF- $\beta 1$ may induce the upregulation of SHH expression along with the cytoplasmic accumulation and nuclear translocation of glioma-associated antigen-1 (Gli1). Moreover, the genetic knockout or pharma- 
A

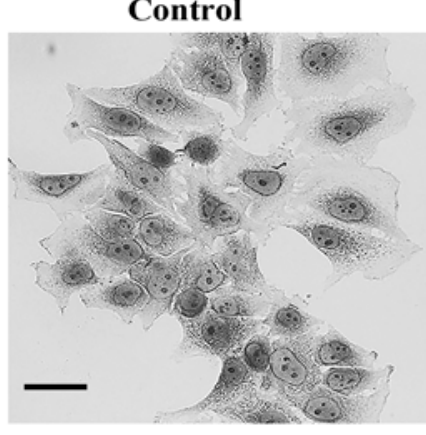

C

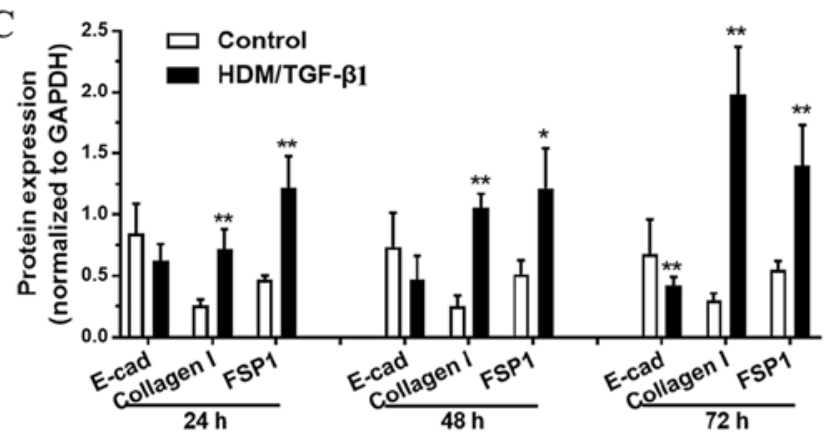

E

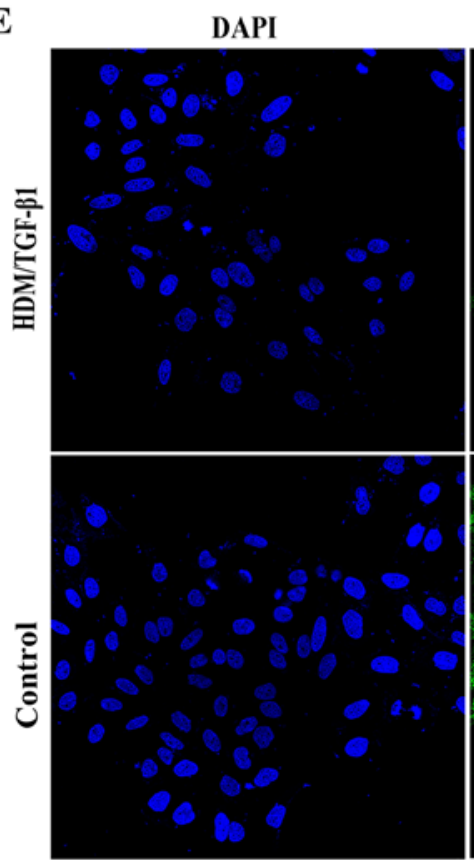

HDM/TGF- 11

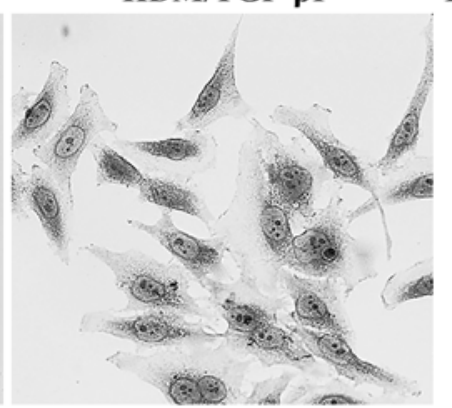

\begin{abstract}
E-cadherin
\end{abstract}

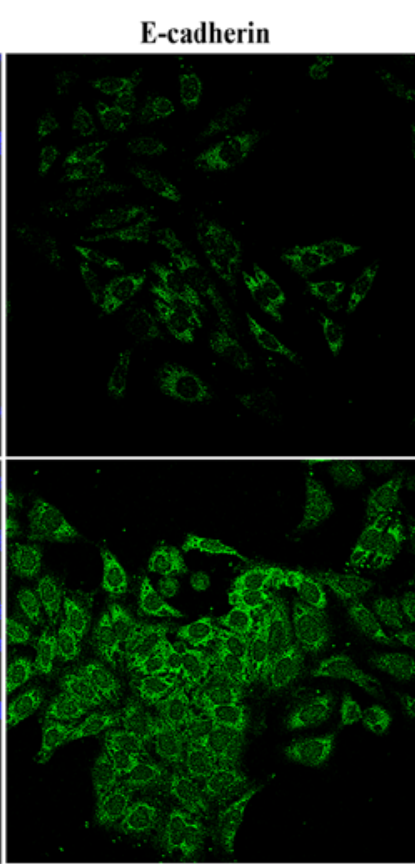

B $\square$ Cyclopamine concentration

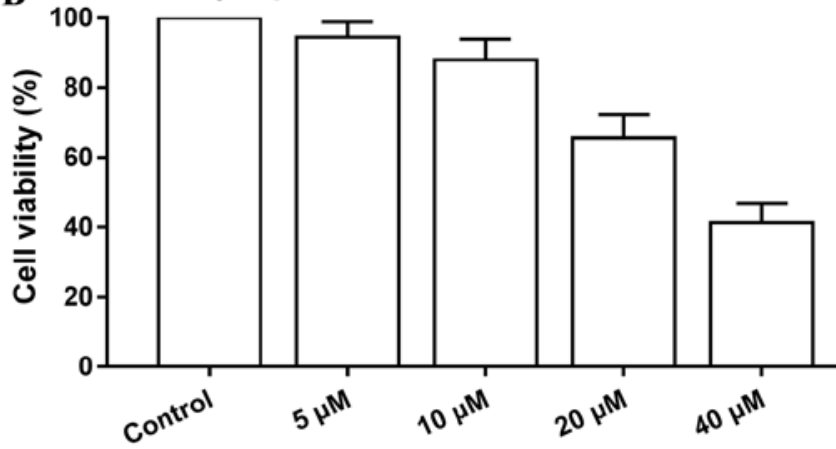

D

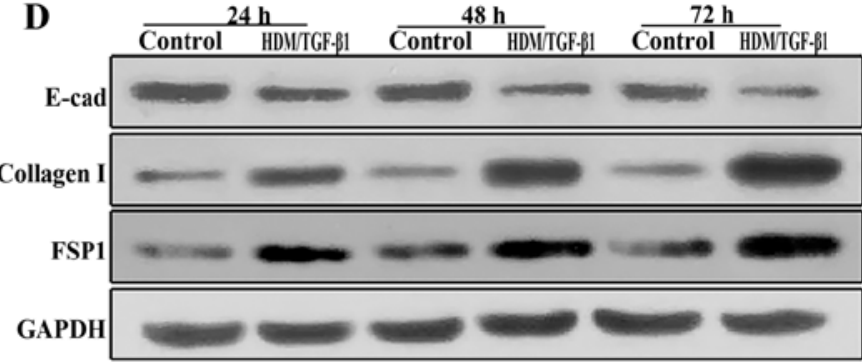

FSP1

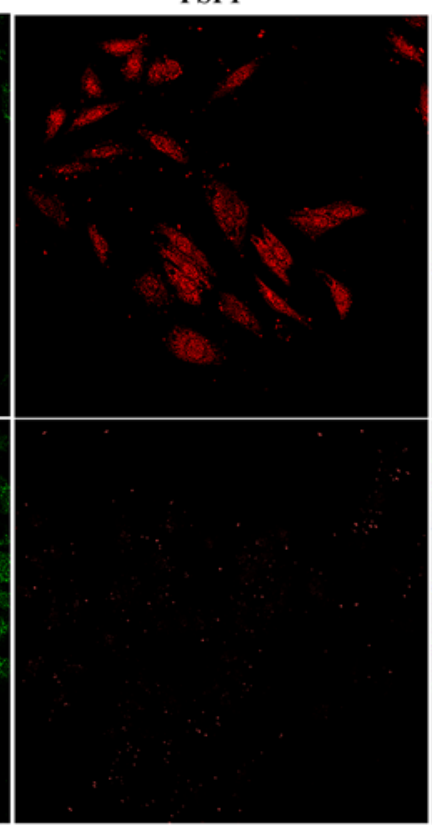

Merged

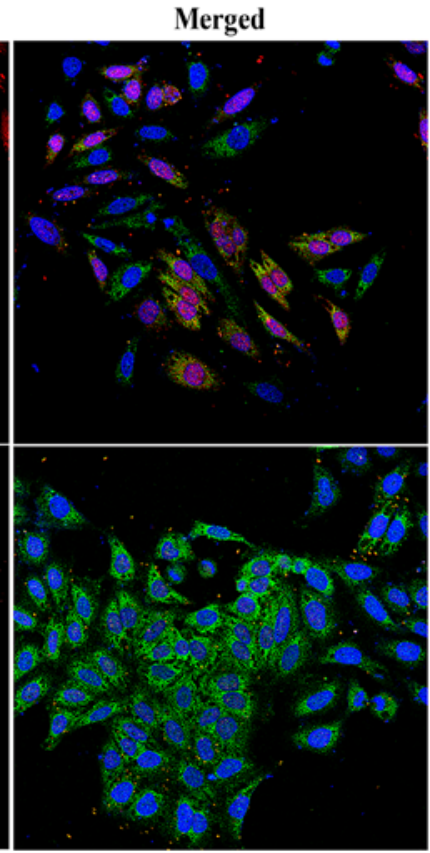

Figure 1. HDM combined with TGF- $\beta 1$ induces EMT in HBECs. (A) HDM combined with TGF- $\beta 1$ induced a morphological phenotype that was characteristic of EMT. Scale bar, $20 \mu \mathrm{m}$. (B) Cyclopamine treatment was shown to lead to the inhibition of 16HBEC survival in a dose-dependent manner using a CCK-8 assay. (C and D) Cells (16HBECs) were stimulated with HDM (30 $\mu \mathrm{g} / \mathrm{ml})$ and TGF- $\beta 1(5 \mathrm{ng} / \mathrm{ml})$ or in complete medium for $24-72 \mathrm{~h}$. Western blotting demonstrated that HDM/TGF- $\beta 1$ treatment increased the expression of mesenchymal markers (collagen I and FSP1) and reduced the expression of an epithelial marker (E-cadherin). An antibody against GAPDH was used as an internal control. ${ }^{*} \mathrm{P}<0.05$ and ${ }^{* *} \mathrm{P}<0.01$ vs. the control. (E) As shown by immunofluorescence staining, the expression of E-cadherin was decreased in the $16 \mathrm{HBECs}$ after $72 \mathrm{~h}$ of treatment with HDM/TGF- $\beta 1$, and the expression of FSP1 increased. Magnification, x400. HDM, house dust mite; TGF- $\beta 1$, transforming growth factor $\beta 1$; EMT, epithelial-mesenchymal transition; HBECs, human bronchial epithelial cells.

cological antagonism of Glil ameliorated EMT. In general, these findings suggest that HDM/TGF- $\beta 1$ may trigger the induction of EMT in HBECs via an SHH signaling mechanism. The inhibition of SHH signaling may be a new therapeutic method for preventing airway remodeling in asthma.
Is EMT important in lung disease? EMT is estimated to contribute $36 \%$ of the fibroblasts in fibrotic lesions (23). In vitro, the stimulation of the bronchial airway epithelium by HDM synergized with TGF- $\beta 1$ and resulted in reduced E-cadherin expression (7). Johnson and colleagues (8) described airway epithelial cell transdifferentiation in a 

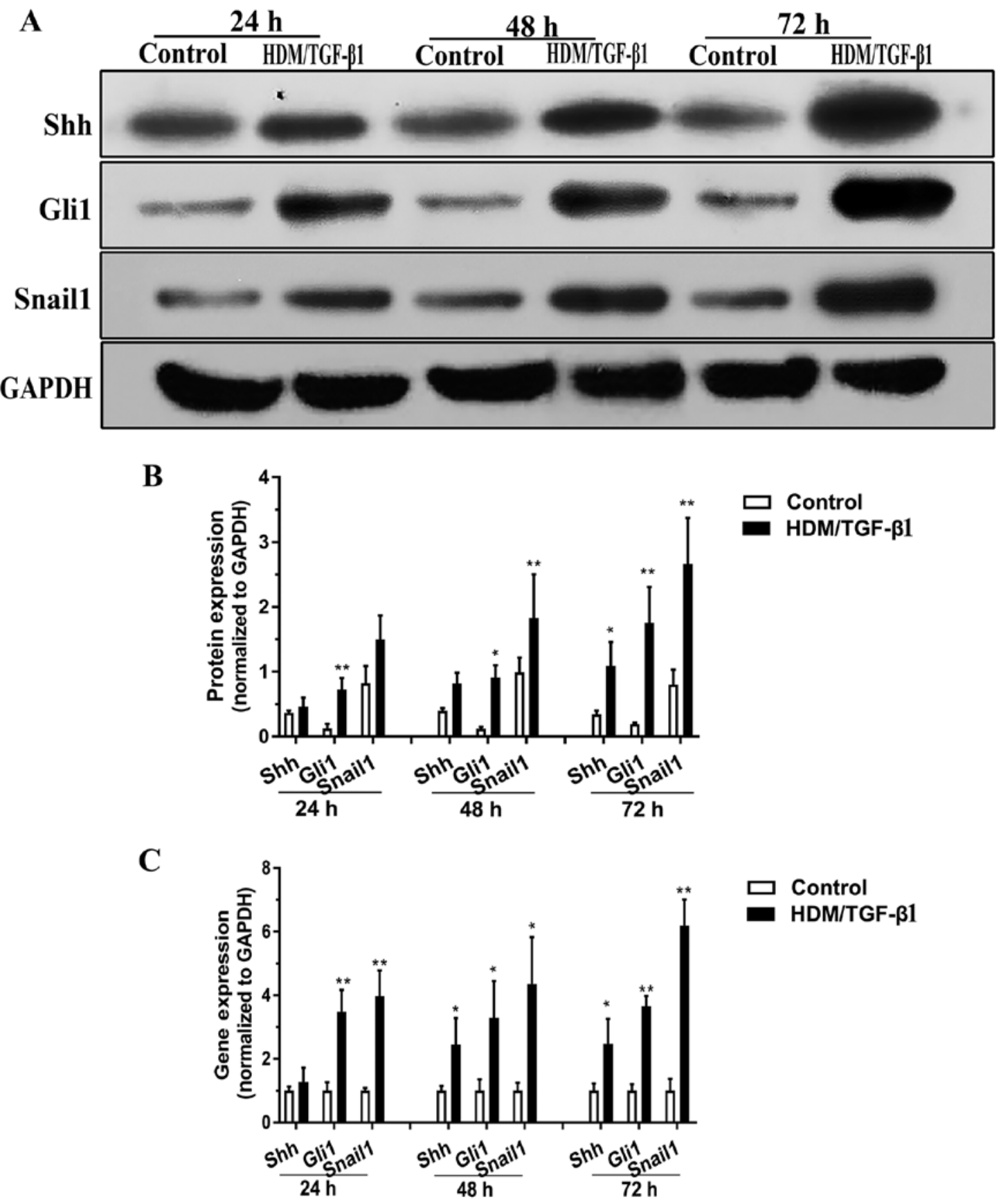

Figure 2. Upregulation of the SHH signaling pathway in 16HBECs stimulated by HDM/TGF- $\beta 1$ for $72 \mathrm{~h}$. (A and B) Western blotting analyses demonstrated that the SHH signaling pathway was induced in 16HBECs by HDM/TGF- $\beta 1$ treatment for 24-72 h. A representation blot and the quantification are shown. GAPDH was used as an internal control. ${ }^{*} \mathrm{P}<0.05$ and ${ }^{* *} \mathrm{P}<0.01$ vs. the control $(\mathrm{n}=3)$. (C) mRNA levels of the members of the SHH signaling pathway at different time points in 16HBECs following treatment with HDM/TGF- $\beta 1$. All values were normalized to GAPDH. ${ }^{*} \mathrm{P}<0.05$ and ${ }^{* * *} \mathrm{P}<0.01 \mathrm{vs}$. control ( $\mathrm{n}=3$ ). SHH, Sonic hedgehog; HBECs, human bronchial epithelial cells; HDM, house dust mite; TGF- $\beta 1$, transforming growth factor $\beta 1$.

mouse model of allergic asthma. In our study, HBECs stimulated by HDM/TGF- $\beta 1$ exhibited morphological features of EMT; by immunofluorescence staining, the expression of an epithelial marker was decreased, while expression of mesenchymal markers was increased compared to those in the controls. The exposure of HBECs to HDM/TGF- $\beta 1$ induced EMT. These findings are in line with a previous study that was performed in an HDM-challenged mouse model (24). This study showed that EMT may contribute to asthma, but the role of EMT in asthma needs to be further studied in the future.
The Hedgehog signaling pathway plays an important role during vertebrate embryonic development and tumorigenesis. Patched (Ptch) was found to impede Smoothened (Smo) activity when the Hedgehog ligand is lacking and then prevents the activation of Gli (14). In the present study, we first detected the gene and protein expression of the core molecular components of the SHH signaling pathway in HBECs stimulated by allergens. By western blot analysis, we found that Shh and Gli1 secretion was significantly increased in 16HBECs exposed to $\mathrm{HDM} / \mathrm{TGF}-\beta 1$ for $48 \mathrm{~h}$ compared to their secretion in control cells, and real-time PCR revealed the significant upregulation 
A
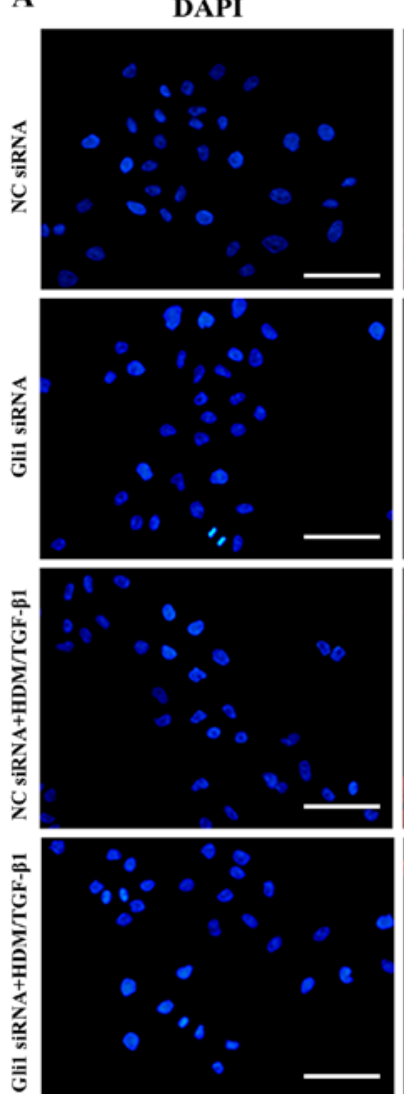

DAPI

B
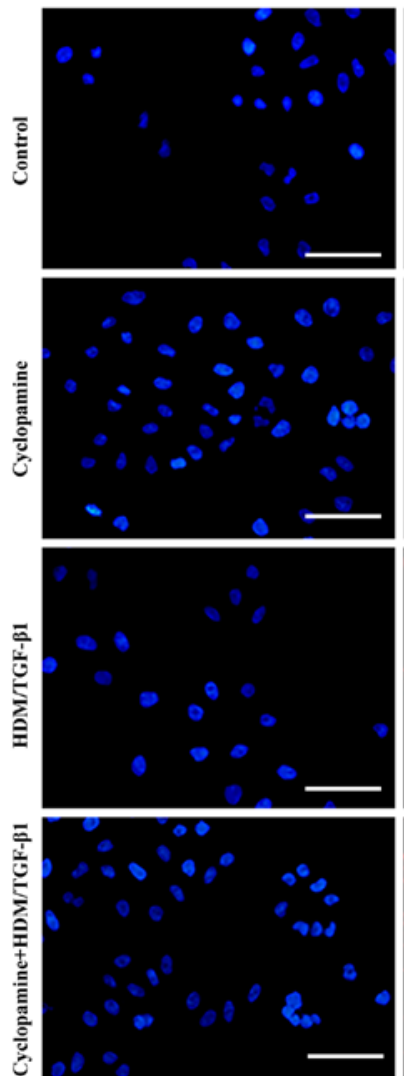

Gli1
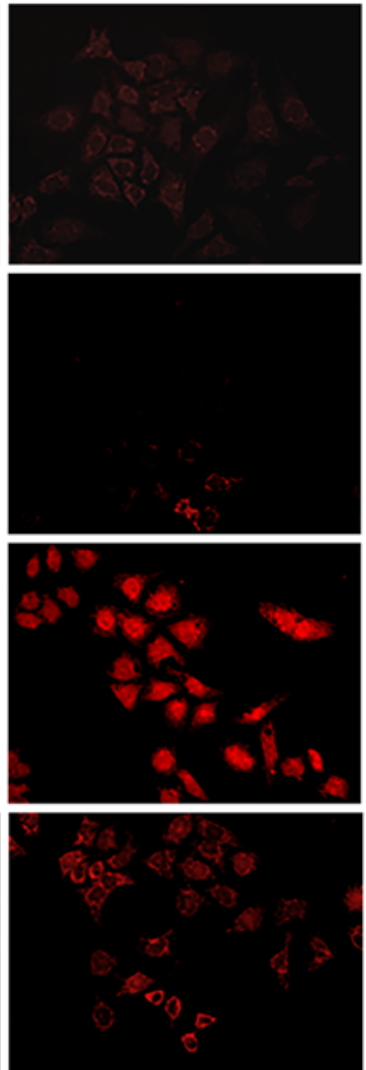

Gli1
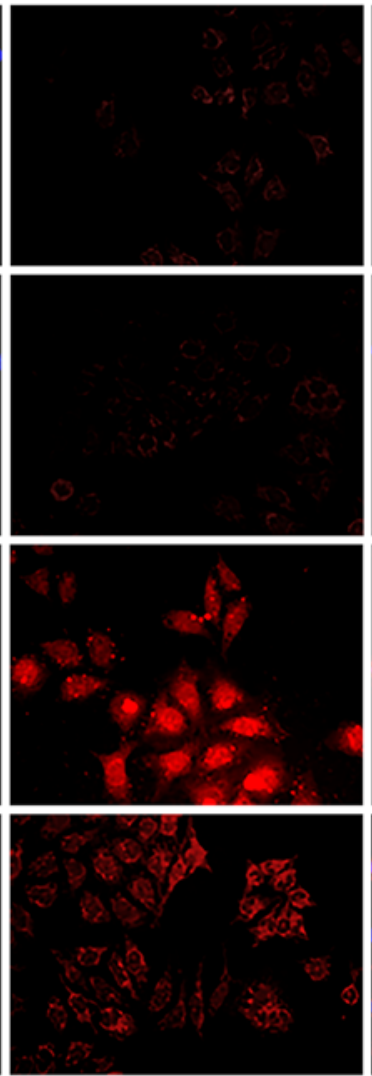

Merged
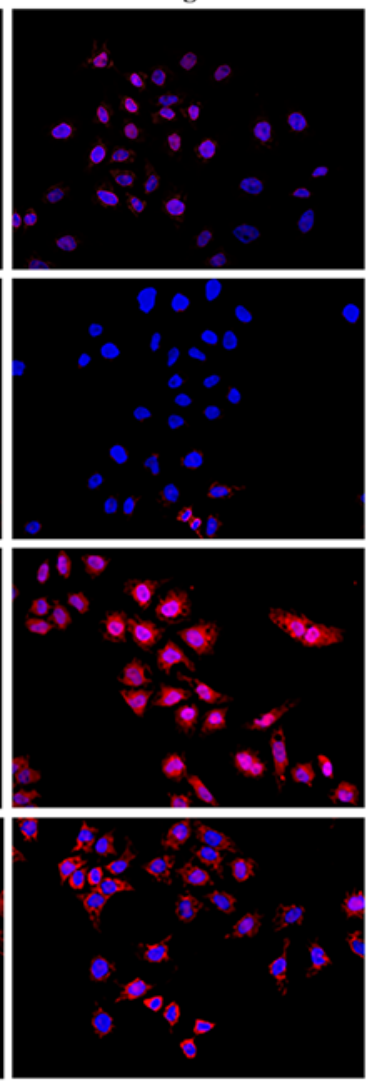

Merged
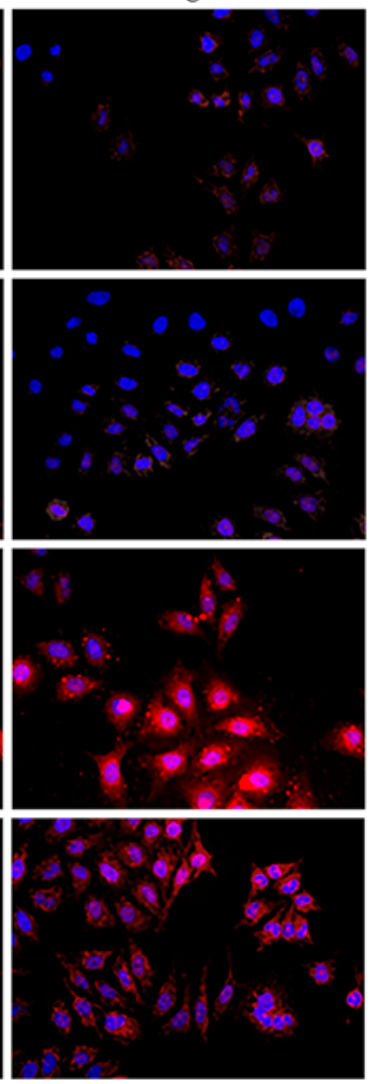

Figure 3. Blockade of SHH signaling in 16HBECs inhibits the HDM/TGF- $\beta 1$-induced nuclear translocation of Gli1. The nuclear translocation of Gli1 was assessed by immunofluorescence staining. Cells were stained with an anti-Gli1 antibody (red) and DAPI (blue). Gli1 protein expression was increased in the treated cells compared to its expression in the control cells, and Gli1 was primarily localized to the nuclei of $16 \mathrm{HBECs}$ stimulated by HDM/TGF- $\beta 1$ for $72 \mathrm{~h}$. After the silencing of Gli1 using siRNA (A) or cyclopamine (B) the nuclear translocation of Gli1 was significantly decreased. A negative control siRNA (NC SiRNA) with no homology to known genes was used as a non-targeting control siRNA. Scale bar, $10 \mu \mathrm{m}$. SHH, Sonic hedgehog; HBECs, human bronchial epithelial cells; HDM, house dust mite; TGF- $\beta 1$, transforming growth factor $\beta 1$; Gli1, glioma-associated antigen-1; HBECs, human bronchial epithelial cells. 
A

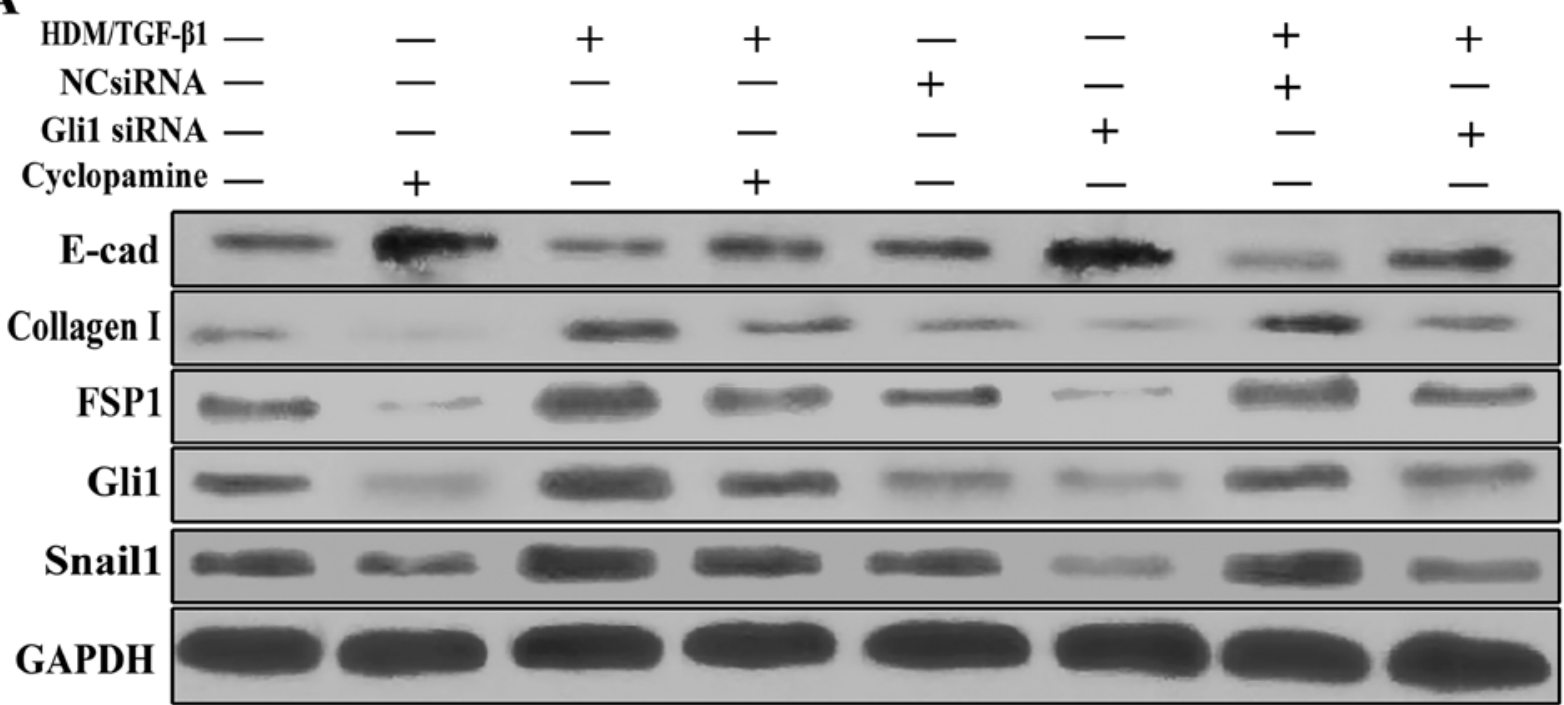

B

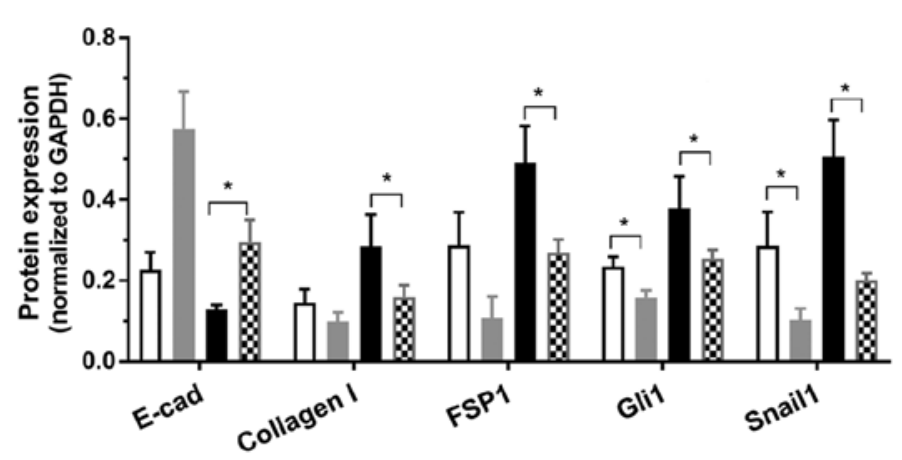

$\square$ NC siRNA

- HDM/TGF- $\beta 1$ + NC siRNA

C
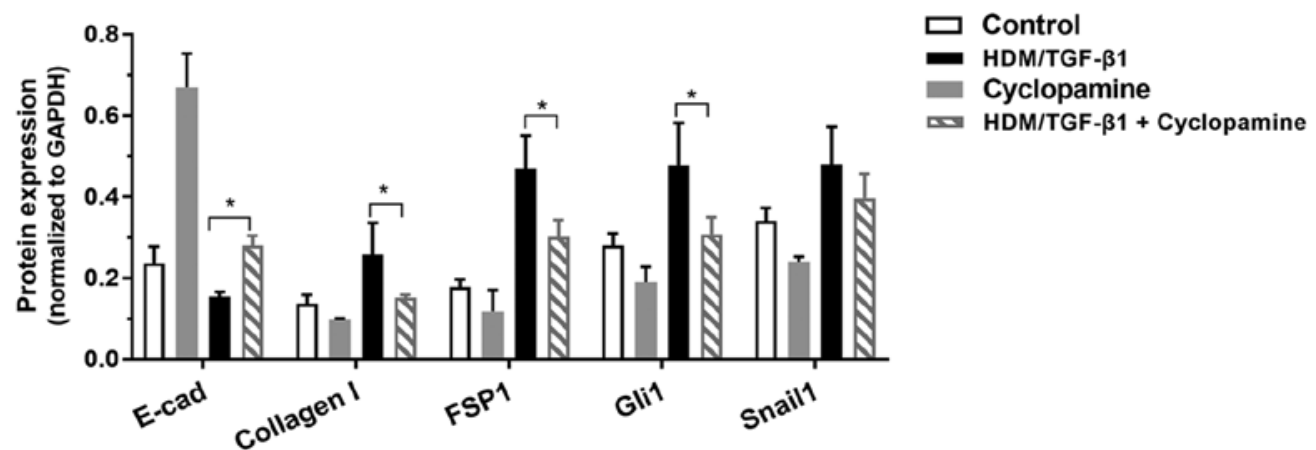

Figure 4. Blockade of SHH signaling reduces HDM/TGF- $\beta 1$-mediated EMT in 16HBECs. Cells (16HBECs) were stimulated with HDM/TGF- $\beta 1$ in the absence or presence of a Gli1 siRNA or cyclopamine for $72 \mathrm{~h}$, as indicated. Western blotting (A) and quantification (B) showed that the transfection of cells with a Gli1-specific siRNA inhibited the expression of SHH signaling proteins (Gli1 and Snail1) and suppressed HDM/TGF- $\beta 1$-induced EMT changes (increased expression of E-cadherin and decreased expression of collagen I and FSP1). No antagonistic effect was detected in 16HBECs transfected with a control siRNA. The western blotting (A) and quantification (C) demonstrated that cyclopamine inhibited SHH signaling and attenuated HDM/TGF- $\beta 1$-induced EMT. "P<0.05 $(\mathrm{n}=3)$. SHH, Sonic hedgehog; HDM, house dust mite; TGF- $\beta 1$, transforming growth factor $\beta 1$; EMT, epithelial-mesenchymal transition; HBECs, human bronchial epithelial cells; Gli1, glioma-associated antigen-1.

of SHH and Gli1 mRNA expression. Our results suggested that the gene expression and protein levels of Shh/Gli1 were upregulated in response to HDM/TGF- $\beta 1$. By immunofluorescence staining, we found that Gli1 protein expression was increased, and Gli1 was localized primarily to the nucleus of 16HBECs treated with HDM/TGF- $\beta 1$. The nuclear localization and accumulation of Gli1 is essential to its transcriptional activity. The efficiency of Gli1 siRNA or cyclopamine in the inhibition of Glil expression was reported in a previous study (18). After silencing of Gli1 using siRNA or cyclopamine, the nuclear translocation and protein expression of Gli1 were significantly decreased. These findings demonstrated that SHH signaling may play a role in asthma. Our findings are consistent with two previous studies that showed that SHH was significantly increased in the bronchial epithelium in asthma patients $(11,25)$. A recent study reported that Gli protein expression was strongly associated with EMT biomarkers in lung carcinomas (26). 
In the present study, the expression of the Snaill protein was significantly increased in $16 \mathrm{HBEC}$ exposed to HDM/TGF- $\beta 1$. Some studies have emphasized the important roles of the transcription factor Snail1 during the EMT process (27). To examine the role of Shh/Gli1 in HDM/TGF- $\beta 1$-induced Snail 1 activation and the EMT process, we treated HBECs with Gli1 siRNA or cyclopamine. Following the blockade of SHH signaling, 16HBECs stimulated by HDM/TGF- $\beta 1$ had lower cytoplasmic Snaill expression as well as reduced EMT biomarker alterations than untreated cells. Based on these data, we proposed that the combination of HDM and TGF- $\beta 1$ activated Shh/Gli1, which upregulated the nuclear translocation of Gli1 and in turn increased Snaill expression, finally resulting in EMT.

If SHH signaling is important for EMT induction, the inhibition of SHH signaling may have antifibrotic effects. The use of cyclopamine, a well-characterized Smo inhibitor, is limited in vivo due to its short half-life and off-target effects occur at high doses (28). Ding et al (18) demonstrated that cyclopamine could prevent fibroblast proliferation and matrix synthesis in renal tubulointerstitial fibrosis. The present study showed that cyclopamine antagonized the EMT process and reduced ECM production in vitro. However, the antifibrotic effects of cyclopamine in asthma warrant further study.

In summary, these findings demonstrate that the combination of HDM and TGF- $\beta 1$ may induce EMT in HBECs. The effect was mainly triggered by the $\mathrm{SHH}$ signaling pathway, and the inhibition of SHH signaling abolished SHH-induced EMT and ECM synthesis. Therefore, SHH signaling may play a key role in EMT and airway remodeling in asthma, and pharmacological antagonism of $\mathrm{SHH}$ signaling may have a therapeutic effect in asthma. In the future, the role of the in vivo blockade of Shh/Gli1 signaling during EMT requires further study.

\section{Acknowledgements}

Not applicable.

\section{Funding}

This study was supported by Zhejiang Provincial Natural Science Foundation of China (grant no. LY16H010002, 2016).

\section{Availability of data and materials}

The datasets used and/or analyzed during the current study are available from the corresponding author on reasonable request.

\section{Authors' contributions}

YZ and WH designed the research study. YZ, WH, LZ and YM performed the experiments, WS and YM analyzed the data and prepared the figures. YZ drafted the manuscript. YZ and YM edited and revised manuscript. YZ and WH interpreted the results of the experiments. All authors read and approved the manuscript and agree to be accountable for all aspects of the research in ensuring that the accuracy or integrity of any part of the work are appropriately investigated and resolved.

\section{Ethics approval and consent to participate}

Not applicable.

\section{Patient consent for publication}

Not applicable.

\section{Competing interests}

The authors declare that they have no competing interests.

\section{References}

1. Broide DH: Immunologic and inflammatory mechanisms that drive asthma progression to remodeling. J Allergy Clin Immunol 121: 560-572, 2008

2. Holgate ST, Davies DE, Puddicombe S, Richter A, Lackie P, Lordan $\mathbf{J}$ and Howarth P: Mechanisms of airway epithelial damage: Epithelial-mesenchymal interactions in the pathogenesis of asthma. Eur Respir J Suppl 44: 24S-29S, 2003.

3. Davies DE: The role of the epithelium in airway remodeling in asthma. Proc Am Thorac Soc 6: 678-682, 2009.

4. Pain M, Bermudez O, Lacoste P, Royer PJ, Botturi K, Tissot A, Brouard S, Eickelberg $\mathrm{O}$ and Magnan A: Tissue remodelling in chronic bronchial diseases: From the epithelial to mesenchymal phenotype. Eur Respir Rev 23: 118-130, 2014

5. Borthwick LA, Parker SM, Brougham KA, Johnson GE, Gorowiec MR, Ward C, Lordan JL, Corris PA, Kirby JA and Fisher AJ: Epithelial to mesenchymal transition (EMT) and airway remodelling after human lung transplantation. Thorax 64 : 770-777, 2009.

6. Han Q, Lin L, Zhao B, Wang N and Liu X: Inhibition of mTOR ameliorates bleomycin-induced pulmonary fibrosis by regulating epithelial-mesenchymal transition. Biochem Biophys Res Commun 500: 839-845, 2018.

7. Heijink IH, Postma DS, Noordhoek JA, Broekema M and Kapus A: House dust mite-promoted epithelial-to-mesenchymal transition in human bronchial epithelium. Am J Respir Cell Mol Biol 42: 69-79, 2010.

8. Johnson JR, Roos A, Berg T, Nord M and Fuxe J: Chronic respiratory aeroallergen exposure in mice induces epithelialmesenchymal transition in the large airways. PLoS One 6: e16175, 2011.

9. Choudhry Z, Rikani AA, Choudhry AM, Tariq S, Zakaria F, Asghar MW, Sarfraz MK, Haider K, Shafiq AA and Mobassarah NJ: Sonic hedgehog signalling pathway: A complex network. Ann Neurosci 21: 28-31, 2014.

10. Rimkus TK, Carpenter RL, Qasem S, Chan M and Lo HW: Targeting the Sonic Hedgehog Signaling Pathway: Review of Smoothened and GLI Inhibitors. Cancers (Basel) 8: pii: E22, 2016.

11. Xu C, Zou C, Hussain M, Shi W, Shao Y, Jiang Z, Wu X, Lu M, $\mathrm{Wu}$ J, Xie Q, et al: High expression of Sonic hedgehog in allergic airway epithelia contributes to goblet cell metaplasia. Mucosal Immunol 11: 1306-1315, 2018.

12. Thiery JP, Acloque H, Huang RY and Nieto MA: Epithelialmesenchymal transitions in development and disease. Cell 139: 871-890, 2009.

13. Watkins DN, Berman DM, Burkholder SG, Wang B, Beachy PA and Baylin SB: Hedgehog signalling within airway epithelial progenitors and in small-cell lung cancer. Nature 422: 313-317, 2003.

14. Kugler MC, Joyner AL, Loomis CA and Munger JS: Sonic hedgehog signaling in the lung. From development to disease. Am J Respir Cell Mol Biol 52: 1-13, 2015.

15. Zou W, Zou Y, Zhao Z, Li B and Ran P: Nicotine-induced epithelial-mesenchymal transition via Wnt/ $\beta$-catenin signaling in human airway epithelial cells. Am J Physiol Lung Cell Mol Physiol 304: L199-L209, 2013.

16. Livak KJ and Schmittgen TD: Analysis of relative gene expression data using real-time quantitative PCR and the 2(-Delta Delta C(T)) method. Methods 25: 402-408, 2001.

17. Ahmad A, Maitah MY, Ginnebaugh KR, Li Y, Bao B, Gadgeel SM and Sarkar FH: Inhibition of Hedgehog signaling sensitizes NSCLC cells to standard therapies through modulation of EMT-regulating miRNAs. J Hematol Oncol 6: 77, 2013. 
18. Ding H, Zhou D, Hao S, Zhou L, He W, Nie J, Hou FF and Liu Y: Sonic hedgehog signaling mediates epithelial-mesenchymal communication and promotes renal fibrosis. J Am Soc Nephrol 23: 801-813, 2012.

19. Steelant B, Farre R, Wawrzyniak P, Belmans J, Dekimpe E, Vanheel H, Van Gerven L, Kortekaas Krohn I, Bullens DMA, Ceuppens JL, et al: Impaired barrier function in patients with house dust mite-induced allergic rhinitis is accompanied by decreased occludin and zonula occludens-1 expression. J Allergy Clin Immunol 137: 1043-1053.e5, 2016.

20. Bolanos AL, Milla CM, Lira JC, Ramírez R, Checa M, Barrera L, García-Alvarez J, Carbajal V, Becerril C, Gaxiola M, et al: Role of sonic hedgehog in idiopathic pulmonary fibrosis. Am J Physiol Lung Cell Mol Physiol 303: L978-L990, 2012.

21. Grindley JC, Bellusci S, Perkins D and Hogan BL: Evidence for the involvement of the Gli gene family in embryonic mouse lung development. Dev Biol 188: 337-348, 1997.

22. Zavadil J and Bottinger EP: TGF-beta and epithelial-to-mesenchymal transitions. Oncogene 24: 5764-5774, 2005.

23. Iwano M, Plieth D, Danoff TM, Xue C, Okada H and Neilson EG: Evidence that fibroblasts derive from epithelium during tissue fibrosis. J Clin Invest 110: 341-350, 2002.

24. Pu Y, Liu Y, Liao S, Miao S, Zhou L and Wan L: Azithromycin ameliorates OVA-induced airway remodeling in Balb/c mice via suppression of epithelial-to-mesenchymal transition. Int Immunopharmacol 58: 87-93, 2018.
25. Standing ASI, Yanez DC, Ross R, Crompton T and Furmanski AL: Frontline Science: Shh production and Gli signaling is activated in vivo in lung, enhancing the Th2 response during a murine model of allergic asthma. J Leukoc Biol 102: 965-976, 2017.

26. Yue D, Li H, Che J, Zhang Y, Tseng HH, Jin JQ, Luh TM, Giroux-Leprieur E, Mo M, Zheng Q, et al: Hedgehog/Gli promotes epithelial-mesenchymal transition in lung squamous cell carcinomas. J Exp Clin Cancer Res 33: 34, 2014

27. Hills CE and Squires PE: TGF-beta1-induced epithelial-to-mesenchymal transition and therapeutic intervention in diabetic nephropathy. Am J Nephrol 31: 68-74, 2010.

28. Zhao C, Chen A, Jamieson CH, Fereshteh M, Abrahamsson A, Blum J, Kwon HY, Kim J, Chute JP, Rizzieri D, et al: Hedgehog signalling is essential for maintenance of cancer stem cells in myeloid leukaemia. Nature 458: 776-779, 2009.

(i)(3) This work is licensed under a Creative Common Attribution-NonCommercial-NoDerivatives 4.0 International (CC BY-NC-ND 4.0) License. 